UDC 517.98

\author{
E. Alizadeh, V. SAdri
}

\title{
CONSTRUCTIONS OF WEAVING CONTINUOUS G-FRAMES FOR OPERATORS IN HILBERT SPACES
}

\begin{abstract}
In this paper, we introduce the concept of weaving continuous K-g-frames in Hilbert spaces, which are the generalization of weaving $\mathrm{K}$-g-frames and weaving c-g-frames. We prove some new results for these frames, focusing on the constructions of c-K-g-woven frames for Hilbert spaces by certain operators with specific properties. Finally, we verify a Paley-Wiener type perturbation of these frames.
\end{abstract}

Key words: $c-K$-g-frame, continuous weaving frame, $c$-K-g-woven frames

2010 Mathematical Subject Classification: Primary 42C15; Secondary 42C40, $41 A 58$

1. Introduction and preliminaries. Duffin and Scheaffer introduced frames [13] in the context of non-harmonic Fourier series. Frames are very useful in the characterization of function spaces and fields of applications, such as filter bank theory, signal and image processing, coding, and wireless communications. Nowadays, frames play a significant role in both pure and applied mathematics, thus constituting a fundamental research area in mathematics, computer science, and engineering; however, technical advances and measure amounts of data which cannot be handled with a signal processing system have been increased using the various frames like g-frame [21], fusion frame [7], K-frame [16], and weaving frames [6], etc.

Continuous frames were introduced by Ali, Antoine, and Gazeau [2] and later, independently, by Kaiser [17]. These frames are the first generalization frames to measure spaces. For more studies about these frames, we refer to [14]. By combining the above-mentioned extensions of frames, the new and more general notion of continuous g-frame has been introduced in [11].

(c) Petrozavodsk State University, 2021 
In 2012, Găvruţa introduced new kinds of frames for operators (or K-frames) in [16] while studying the atomic systems with respect to a bounded operator $K$, already introduced by Feichtinger and Werther in [15], who showed that atomic systems for $K$ are equivalent to the K-frames. Note that the continuous case of these frames had previously been introduced in [3], [4]. For another kind of K-frames, we refer to [18-20].

Recently, motivated by a question in distributed signal processing, a new concept of weaving frames has been introduced by Bemrose et al. [6]. In their work, two frames $\left\{f_{j}\right\}_{j \in \mathbb{J}}$ and $\left\{g_{j}\right\}_{j \in \mathbb{J}}$ for a Hilbert space $H$ are (weakly) woven if, for each subset $\sigma \in \mathbb{J}$, the family $\left\{f_{j}\right\}_{j \in \sigma} \cup\left\{g_{j}\right\}_{j \in \sigma^{c}}$ is a frame for $H$.

In this paper, we generalize some results in [5] to the continuous version of weaving c-g-frames for operators in Hilbert spaces.

Throughout the paper, $(\Omega, \mu)$ is a measure space with positive measure $\mu, H$ and $\left\{H_{\omega}\right\}_{\omega \in \Omega}$ are Hilbert spaces and a family of Hilbert spaces, respectively, and $\mathcal{B}(H, K)$ is the set of all bounded and linear operators from $H$ to $K$. If $H=K$, then $\mathcal{B}(H, H)$ are denoted by $\mathcal{B}(H)$ and $K \in \mathcal{B}(H)$. For each $m>1$ where $m \in \mathbb{N}$, we define $[m]:=\{1,2, \ldots, m\}$ and $[m]^{c}=\{m+1, m+2, \ldots\}$.

First, we need the pseudo-inverse operator. If an operator $U$ has closed range, then there exists a right-inverse operator $U^{\dagger}$ (pseudo-inverse of $U$ ) in the following sense (see [9]):

Lemma 1. Let $U \in \mathcal{B}(K, H)$ be a bounded operator with closed range $\mathcal{R}(U)$. Then there exists a bounded operator $U^{\dagger} \in \mathcal{B}(H, K)$ for which

$$
U U^{\dagger} x=x, \quad x \in \mathcal{R}(U) .
$$

Moreover, the orthogonal projection of $H$ onto $\mathcal{R}(U)$ is given by $U U^{\dagger}$.

Lemma 2. [12] Let $L_{1} \in \mathcal{B}\left(H_{1}, H\right)$ and $L_{2} \in \mathcal{B}\left(H_{2}, H\right)$. Then the following assertions are equivalent:

1) $\mathcal{R}\left(L_{1}\right) \subseteq \mathcal{R}\left(L_{2}\right)$;

2) $L_{1} L_{1}^{*} \leqslant \lambda^{2} L_{2} L_{2}^{*}$ for some $\lambda>0$;

3) there exists a mapping $U \in \mathcal{B}\left(H_{1}, H_{2}\right)$, such that $L_{1}=L_{2} U$.

We first mention the definition of the continuous K-frame from [18].

Definition 1. Suppose that $(\Omega, \mu)$ is a measure space with positive measure $\mu$ and $K \in \mathcal{B}(H)$. A mapping $F: \Omega \rightarrow H$ is called a continuous $K$-frame (or c-K-frame) for $H$ with respect to $(\Omega, \mu)$, if 
(i) For all $h \in H, \omega \mapsto\langle h, F(\omega)\rangle$ is a measurable function on $\Omega$,

(ii) there exist positive constants $A$ and $B$, such that for each $f \in H$,

$$
A\left\|K^{*} h\right\|^{2} \leqslant \int_{\Omega}|\langle h, F(\omega)\rangle|^{2} d \mu(x) \leqslant B\|h\|^{2} .
$$

When $K=I d_{H}$, we get the concept continuous frame (or c-frame) via [14].

Now, we summarize some facts about c-g-frames from [1]. Define

$$
\Pi_{\omega \in \Omega} H_{\omega}=\left\{F: \Omega \longrightarrow \cup_{\omega \in \Omega} H_{\omega}: F(\omega) \in H_{\omega}\right\} .
$$

We say that $F \in \Pi_{\omega \in \Omega} H_{\omega}$ is strongly measurable if $F$, as a mapping of $\Omega$ to $\oplus_{\omega \in \Omega} H_{\omega}$, is measurable. Let $\mathfrak{L}^{2}\left(H_{\omega}, \mu\right)=\left\{F \in \Pi_{\omega \in \Omega} H_{\omega}: F\right.$ be strongly measurable, $\left.\int_{\Omega}\|F(\omega)\|^{2} d \mu(\omega)<\infty\right\}$.

With the inner product given by

$$
\langle F, G\rangle=\int_{\Omega}\langle F(\omega), G(\omega)\rangle d \mu(\omega) .
$$

It can be proved that $\mathfrak{L}^{2}\left(H_{\omega}, \mu\right)$ is a Hilbert space [1]. We will denote the norm of $F \in \mathfrak{L}^{2}\left(H_{\omega}, \mu\right)$ by $\|F\|_{2}$.

The continuous version of K-g-frames was introduced in [3] in the following way.

Definition 2. [3] Let $K \in \mathcal{B}(H)$. A family $\left\{\Lambda_{\omega} \in \mathcal{B}\left(H, H_{\omega}\right)\right\}_{\omega \in \Omega}$ is called a continuous $K$ - $g$-frame (or briefly $c$ - $K$ - $g$-frame) for $H$ with respect to $\left\{H_{\omega}\right\}_{\omega \in \Omega}$, if

(i) the mapping $\Omega \longmapsto \mathbb{C}, \omega \longmapsto\left\|\Lambda_{\omega} f\right\|$ is measurable for any $f \in H$.

(ii) there exist constants $0<A \leqslant B<\infty$, such that for each $f \in H$ :

$$
A\left\|K^{*} f\right\|^{2} \leqslant \int_{\Omega}\left\|\Lambda_{\omega} f\right\|^{2} d \mu(\omega) \leqslant B\|f\|^{2} .
$$

A family $\left\{\Lambda_{\omega}\right\}_{\omega \in \Omega}$ is called a c-g-Bessel family if the right-hand inequality (2) holds; the number $B$ is called the Bessel constant. If $K=I d_{H}$, then the family $\left\{\Lambda_{\omega}\right\}_{\omega \in \Omega}$ is called c-g-frame. If $A, B$ can be chosen such 
that $A=B$, then $\left\{\Lambda_{\omega}\right\}_{\omega \in \Omega}$ is called a tight c-K-g-frame and if $A=B=1$, it is called Parseval c-K-g-frame, and we have

$$
\int_{\Omega}\left\|\Lambda_{\omega} f\right\|^{2} d \mu(\omega)=\left\|K^{*} f\right\|^{2} .
$$

In all kinds of $\mathrm{K}$-frames the synthesis and analysis operators are the same; so if $\left\{\Lambda_{\omega}\right\}_{\omega \in \Omega}$ is a continuous g-Bessel family for $H$ with respect to $\left\{H_{\omega}\right\}_{\omega \in \Omega}$ with the bound $B$, then these operators are defined by (for more details, we refer to [3])

$$
\begin{aligned}
T_{\Lambda} & : \mathfrak{L}^{2}\left(H_{\omega}, \mu\right) \longrightarrow H, \\
\left\langle T_{\Lambda} F, g\right\rangle & =\int_{\Omega}\left\langle\Lambda_{\omega}^{*} F(\omega), g\right\rangle d \mu(\omega),
\end{aligned}
$$

and

$$
\begin{aligned}
& T_{\Lambda}^{*}: H \longrightarrow \mathfrak{L}^{2}\left(H_{\omega}, \mu\right) \\
& T_{\Lambda}^{*}(g)(\omega)=\Lambda_{\omega} g .
\end{aligned}
$$

Thus, the frame operator $S_{\Lambda}=T_{\Lambda} T_{\Lambda}^{*}$ is given by

$$
S_{\Lambda}: H \longrightarrow H, \quad\left\langle S_{\Lambda} f, g\right\rangle=\int_{\Omega}\left\langle f, \Lambda_{\omega} \Lambda_{\omega}^{*} g\right\rangle d \mu .
$$

Therefore,

$$
A K K^{*} \leqslant S_{\Lambda} \leqslant B I d_{H}
$$

Definition 3. [22] A family of c-frames $\left\{F_{\omega i}\right\}_{\omega \in \Omega, i \in[m]}$ for $H$ with respect to $\mu$ is said to be c-woven, if there exist universal same positive constants $0<A \leqslant B<\infty$, such that for each partition $\left\{\sigma_{i}\right\}_{i \in[m]}$ of $\Omega$, the family $\left\{F_{\omega i}\right\}_{\omega \in \sigma_{i}, i \in[m]}$ is a c-frame for $H$ with the bounds $A$ and $B$. Each family $\left\{F_{\omega i}\right\}_{\omega \in \sigma_{i}, i \in[m]}$ is called a weaving.

2. Weaving Continuous K-g-Frames. In this section, we introduce the notion of continuous K-g-woven frames in Hilbert spaces and discuss some of their properties. Throughout the paper, by partition of a measure space $(\Omega, \mu)$ we mean partition of $\Omega$ into disjoint measurable sets.

Definition 4. A family of $c$-K-g-frames $\left\{\Lambda_{\omega i} \in \mathcal{B}\left(H, H_{\omega}\right)\right\}_{\omega \in \Omega, i \in[m]}$ for $H$ is said to be continuous $K$-g-woven (or $c-K-g$-woven) if there exist universal 
constants $0<A \leqslant B$, such that for each partition $\left\{\sigma_{i}\right\}_{i \in[m]}$ of $\Omega$, the family $\left\{\Lambda_{\omega i}\right\}_{\omega \in \sigma_{i}, i \in[m]}$ is a $c$ - $K$-g-frame for $H$ with bounds $A$ and $B$.

In the above definition, $A$ and $B$ are called universal c-K-g-frame bounds. It is easy to show that every c-K-g-woven has a universal upper c-K-g-frame bound. Indeed, let $\left\{\Lambda_{\omega i}\right\}_{\omega \in \Omega}$ be a c-g-Bessel family for $H$ with bound $B_{i}$ for each $i \in[m]$. Then, for any partition $\left\{\sigma_{i}\right\}_{i \in[m]}$ of $\Omega$ and $f \in H$, we have

$$
\sum_{i \in[m]} \int_{\sigma_{i}}\left\|\Lambda_{\omega i} f\right\|^{2} d \mu \leqslant \sum_{i \in[m]} \int_{\Omega}\left\|\Lambda_{\omega i} f\right\|^{2} d \mu \leqslant\left(\sum_{i \in[m]} B_{i}\right)\|f\|^{2} .
$$

The next proposition shows that it is enough to check c-K-g-weaving on a smaller measurable space than the original. It is an extension of Proposition 3.10 in [22].

Theorem 1. For each $i \in[m]$, let $\left\{\Lambda_{\omega i}\right\}_{\omega \in \Omega}$ be a $c-K$-g-frame for $H$ with frame bounds $A_{i}$ and $B_{i}$. If there exists a measurable subset $\Sigma \subset \Omega$, such that the family of $c-K$-g-frame $\left\{\Lambda_{\omega i}\right\}_{\omega \in \Sigma, i \in[m]}$ is a $c-K-g$-woven for $H$ with universal frame bounds $A$ and $B$, then $\left\{\Lambda_{\omega i}\right\}_{\omega \in \Omega, i \in[m]}$ is a $c-K$-g-woven for $H$ with universal bounds $A$ and $\sum_{i \in[m]} B_{i}$.

Proof. Suppose that $\left\{\sigma_{i}\right\}_{i \in[m]}$ is a partition of $\Omega$ and $f \in H$. We have

$$
\sum_{i \in[m]} \int_{\sigma_{i}}\left\|\Lambda_{\omega i} f\right\|^{2} d \mu(\omega) \leqslant \sum_{i \in[m]} B_{i}\|f\|^{2} .
$$

For the lower bound, it is clear that $\left\{\sigma_{i} \cap \Sigma\right\}_{i \in[m]}$ is a partition of $\Sigma$. Thus, $\left\{\Lambda_{\omega i}\right\}_{\omega \in \sigma_{i} \cap \Sigma, i \in[m]}$ is a c-K-g-frame for $H$ with the lower frame bound $A$. Hence, for each $f \in H$

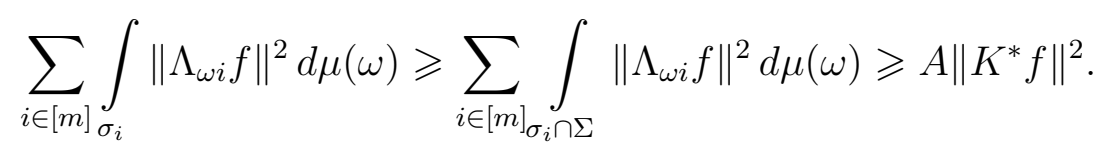

This completes the proof.

Casazza and Lynch in [8] showed that it is possible to remove vectors from woven frames and still be left with woven frames. Later, this topic was presented in [22]. Now, we study it for c-K-g-woven in the following Theorem. 
Theorem 2. Let $\left\{\Lambda_{\omega i}\right\}_{\omega \in \Omega, i \in[m]}$ be a c-K-g-woven for $H$ with universal bounds $A$ and $B$. If there exists $0<D<A$ and a measurable subset $\Sigma \subset \Omega$ and $n \in[m]$, such that for each $f \in H$,

$$
\sum_{i \in[m] \backslash\{n\}_{\Omega \backslash \Sigma}} \int_{\|}\left\|\Lambda_{\omega i} f\right\|^{2} d \mu(\omega) \leqslant D\|f\|^{2}, \quad \forall f \in H,
$$

then the family $\left\{\Lambda_{\omega i}\right\}_{\omega \in \Sigma, i \in[m]}$ is a $c-K$-g-woven for $H$ with frame bounds $A-D$ and $B$.

Proof. Suppose that $\left\{\sigma_{i}\right\}_{i \in[m]}$ is a partition of $\Sigma$ and $\left\{\tau_{i}\right\}_{i \in[m]}$ is a partition of $\Omega \backslash \Sigma$. For a given $f \in H$, define

$$
\varphi: \Sigma \longrightarrow \mathbb{C}, \quad \varphi(\omega)=\sum_{i \in[m]} \chi_{\sigma_{i}}(\omega)\left\|\Lambda_{\omega i} f\right\|,
$$

and

$$
\phi: \Lambda \longrightarrow \mathbb{C}, \quad \phi(\omega)=\sum_{i \in[m]} \chi_{\sigma_{i} \cup \tau_{i}}(\omega)\left\|\Lambda_{\omega i} f\right\|,
$$

where, $\chi_{\sigma_{i}}$ is the characteristic function of $\sigma_{i}$. Since $\left\{\Lambda_{\omega i}\right\}_{\omega \in \sigma_{i} \cup \tau_{i}, i \in[m]}$ is a c-K-g-frame for $H$ and $\varphi=\left.\phi\right|_{\Sigma}$, then $\varphi$ and $\phi$ are measurable. So, for each $f \in H$, we have

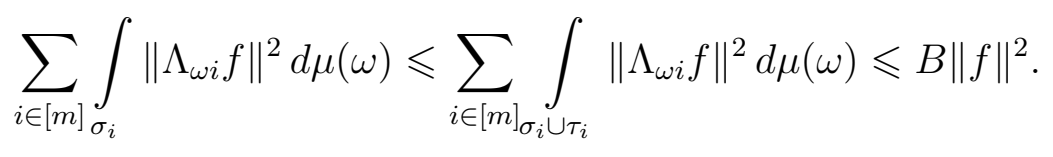

Now, for the lower frame bound, assume that $\left\{\varsigma_{i}\right\}_{i \in[m]}$ is a partition of $\Omega \backslash \Sigma$, such that $\varsigma_{n}=\emptyset$. Then $\left\{\varsigma_{i} \cup \sigma_{i}\right\}_{i \in[m]}$ is a partition of $\Omega$ and, so, for any $f \in H$ we have:

$$
\begin{aligned}
& \sum_{i \in[m]} \int_{\sigma_{i}}\left\|\Lambda_{\omega i} f\right\|^{2} d \mu(\omega)= \\
= & \sum_{i \in[m] \backslash\{n\}}\left(\int_{\varsigma_{i} \cup \sigma_{i}}\left\|\Lambda_{\omega i} f\right\|^{2} d \mu(\omega)-\int_{\varsigma_{i}}\left\|\Lambda_{\omega i} f\right\|^{2} d \mu(\omega)\right)+\int_{\sigma_{n}}\left\|\Lambda_{\omega i} f\right\|^{2} d \mu(\omega) \geqslant \\
\geqslant & \sum_{i \in[m] \backslash\{n\}}\left(\int_{\varsigma_{i} \cup \sigma_{i}}\left\|\Lambda_{\omega i} f\right\|^{2} d \mu(\omega)-\int_{\Omega \backslash \Sigma}\left\|\Lambda_{\omega i} f\right\|^{2} d \mu(\omega)\right)+\int_{\sigma_{n}}\left\|\Lambda_{\omega i} f\right\|^{2} d \mu(\omega)= \\
= & \sum_{i \in[m]} \int_{\varsigma_{i} \cup \sigma_{i}}\left\|\Lambda_{\omega i} f\right\|^{2} d \mu(\omega)-\sum_{i \in[m] \backslash\{n\}} \int_{\Omega \backslash \Sigma}\left\|\Lambda_{\omega i} f\right\|^{2} d \mu(\omega) \geqslant(A-D)\left\|K^{*} f\right\|^{2} .
\end{aligned}
$$


The proof is completed.

The next result provides a necessary and sufficient condition for c-K-g-woven that connects to c-K-woven.

Theorem 3. Let $\Omega_{i} \subseteq \Omega$ be measurable subsets for all $i \in[m]$, and let $F_{i}$ and $G_{i}$ be $c$-frame mappings on $\Omega_{i}$ for $H_{\omega}$ with the pair frame bounds $\left(A_{F_{i}}, B_{F_{i}}\right)$, and $\left(A_{G_{i}}, B_{G_{i}}\right)$, respectively, for each $\omega \in \Omega$. Assume that $\Lambda_{\omega i}, \Theta_{\omega i} \in \mathcal{B}\left(H, \Omega_{i}\right)$ for any $i \in[m]$, such that $\left\{\Lambda_{\omega i}\right\}_{\omega \in \Omega, i \in[m]}$ and $\left\{\Theta_{\omega i}\right\}_{\omega \in \Omega, i \in[m]}$ are strongly measurable. Then the following assertions are equivalent:

(I) $\left\{\Lambda_{\omega i}^{*} F_{i}\right\}_{\omega \in \Omega, i \in[m]}$ and $\left\{\Theta_{\omega i}^{*} G_{i}\right\}_{\omega \in \Omega, i \in[m]}$ are $c-K$-woven for $H$.

(II) $\left\{\Lambda_{\omega i}\right\}_{\omega \in \Omega, i \in[m]}$ and $\left\{\Theta_{\omega i}\right\}_{\omega \in \Omega, i \in[m]}$ are $c-K$-g-woven for $H$.

Proof. $(I) \Rightarrow(I I)$. Assume that $\sigma \subset \Omega$ is a measurable subset and $f \in H$. Let $\left\{\Lambda_{\omega i}^{*} F_{i}\right\}_{\omega \in \Omega, i \in[m]}$ and $\left\{\Theta_{\omega i}^{*} G_{i}\right\}_{\omega \in \Omega, i \in[m]}$ be c-K-woven for $H$ with universal frame bounds $C, D$, and $A=\inf \left\{A_{F_{\omega i}}, A_{G_{\omega i}}\right\}$. We have, for each $i \in[m]$ :

$$
\begin{gathered}
A \int_{\sigma}\left\|\Lambda_{\omega i} f\right\|^{2} d \mu(\omega)+A \int_{\sigma^{c}}\left\|\Theta_{\omega i} f\right\|^{2} d \mu(\omega) \leqslant \\
\leqslant \int_{\sigma} A_{F_{\omega i}}\left\|\Lambda_{\omega i} f\right\|^{2} d \mu(\omega)+\int_{\sigma^{c}} A_{G_{\omega i}}\left\|\Theta_{\omega i} f\right\|^{2} d \mu(\omega) \leqslant \\
\leqslant \int_{\sigma} \int_{\Omega_{i}}\left|\left\langle\Lambda_{\omega i} f, F_{i}(x)\right\rangle\right|^{2} d \mu(x) d \mu(\omega)+\iint_{\sigma^{c}}\left|\left\langle\Theta_{\omega i} f, G_{i}(x)\right\rangle\right|^{2} d \mu(x) d \mu(\omega)= \\
=\int_{\sigma} \int_{\Omega_{i}}\left|\left\langle f, \Lambda_{\omega i}^{*} F_{i}(x)\right\rangle\right|^{2} d \mu(x) d \mu(\omega)+\int_{\sigma^{c}} \int_{\Omega_{i}}\left|\left\langle f, \Theta_{\omega i}^{*} G_{i}(x)\right\rangle\right|^{2} d \mu(x) d \mu(\omega) \leqslant
\end{gathered}
$$

In the same way, we conclude that

$$
\begin{aligned}
& B \int_{\sigma}\left\|\Lambda_{\omega i} f\right\|^{2} d \mu(\omega)+B \int_{\sigma^{c}}\left\|\Theta_{\omega i} f\right\|^{2} d \mu(\omega) \geqslant \\
\geqslant & \int_{\sigma} \int_{\Omega_{i}}\left|\left\langle f, \Lambda_{\omega i}^{*} F_{i}(x)\right\rangle\right|^{2} d \mu(x) d \mu(\omega)+\int_{\sigma^{c}} \int_{\Omega_{i}}\left|\left\langle f, \Theta_{\omega i}^{*} G_{i}(x)\right\rangle\right|^{2} d \mu(x) d \mu(\omega) \geqslant \\
& \geqslant C\left\|K^{*} f\right\|^{2},
\end{aligned}
$$


where $B=\sup \left\{B_{F_{\omega i}}, B_{G_{\omega i}}\right\}$. Thus, we have checked that $\left\{\Lambda_{\omega i}\right\}_{\omega \in \Omega, i \in[m]}$ and $\left\{\Theta_{\omega i}\right\}_{\omega \in \Omega, i \in[m]}$ are c-K-g-woven for $H$ with universal frame bounds $\frac{C}{B}$ and $\frac{D}{A}$.

$(I I) \Rightarrow(I)$. Suppose that $\left\{\Lambda_{\omega i}\right\}_{\omega \in \Omega, i \in[m]}$ and $\left\{\Theta_{\omega i}\right\}_{\omega \in \Omega, i \in[m]}$ are c-K-g-woven for $H$ with universal frame bounds $C$ and $D$. Now, we can write for each $f \in H$ :

$$
\begin{gathered}
\iint_{\sigma}\left|\left\langle f, \Lambda_{\omega i}^{*} F_{i}(x)\right\rangle\right|^{2} d \mu(x) d \mu(\omega)+\int_{\sigma^{c}} \int_{\Omega_{i}}\left|\left\langle f, \Theta_{\omega i}^{*} G_{i}(x)\right\rangle\right|^{2} d \mu(x) d \mu(\omega)= \\
=\iint_{\sigma}\left|\left\langle\Lambda_{\Omega_{i}} f, F_{i}(x)\right\rangle\right|^{2} d \mu(x) d \mu(\omega)+\int_{\sigma^{c}} \int_{\Omega_{i}}\left|\left\langle\Theta_{\omega i} f, G_{i}(x)\right\rangle\right|^{2} d \mu(x) d \mu(\omega) \geqslant \\
\geqslant \int_{\sigma} A_{F_{\omega i}}\left\|\Lambda_{\omega i} f\right\|^{2} d \mu(\omega)+\int_{\sigma^{c}} A_{G_{\omega i}}\left\|\Theta_{\omega i} f\right\|^{2} d \mu(\omega) \geqslant \\
\geqslant A\left(\int_{\sigma}\left\|\Lambda_{\omega i} f\right\|^{2} d \mu(\omega)+\int_{\sigma^{c}}\left\|\Theta_{\omega i} f\right\|^{2} d \mu(\omega)\right) \geqslant A C\left\|K^{*} f\right\|^{2} .
\end{gathered}
$$

Also, we can get

$\iint_{\sigma \Omega_{i}}\left|\left\langle f, \Lambda_{\omega i}^{*} F_{i}(x)\right\rangle\right|^{2} d \mu(x) d \mu(\omega)+\iint_{\sigma c} \int_{\Omega_{i}}\left|\left\langle f, \Theta_{\omega i}^{*} G_{i}(x)\right\rangle\right|^{2} d \mu(x) d \mu(\omega) \leqslant B D\|f\|^{2}$.

So, $\left\{\Lambda_{\omega i}^{*} F_{i}\right\}_{\omega \in \Omega, i \in[m]}$ and $\left\{\Theta_{\omega i}^{*} G_{i}\right\}_{\omega \in \Omega, i \in[m]}$ are c-K-woven for $H$ with universal bounds $A C$ and $B D$.

In the next theorem we give a sufficient condition for c-K-g-woven.

Theorem 4. Let $\left\{\Lambda_{\omega i}\right\}_{\omega \in \Omega}$ be a c-K-g-frame for $H$ with frame bounds $A_{i}$ and $B_{i}$ for each $i \in[m]$. Suppose that there exists $M>0$, such that for all $f \in H, i \neq k \in[m]$ and any measurable subset $\Delta \subset \Omega$ :

$\int_{\Delta}\left\|\left(\Lambda_{\omega i}-\Lambda_{\omega k}\right) f\right\|^{2} d \mu(\omega) \leqslant M \min \left\{\int_{\Delta}\left\|\Lambda_{\omega i} f\right\|^{2} d \mu(\omega), \int_{\Delta}\left\|\Lambda_{\omega k} f\right\|^{2} d \mu(\omega)\right\}$.

Then the family $\left\{\Lambda_{\omega i}\right\}_{\omega \in \Omega, i \in[m]}$ is a $c-K-g$-woven with universal bounds

$$
\frac{A}{(m-1)(M+1)+1} \text { and } B \text {, }
$$


where $A:=\sum_{i \in[m]} A_{i}$ and $B=\sum_{i \in[m]} B_{i}$.

Proof. The upper bound is evident. For the lower bound, suppose that $\left\{\sigma_{i}\right\}_{i \in[m]}$ is a partition of $\Omega$ and $f \in H$. Therefore,

$$
\begin{gathered}
\sum_{i \in[m]} A_{i}\left\|K^{*} f\right\|^{2} \leqslant \sum_{i \in[m]} \int_{\Omega}\left\|\Lambda_{\omega i} f\right\|^{2} d \mu(\omega)=\sum_{i \in[m]} \sum_{j \in[m]} \int_{\sigma_{j}}\left\|\Lambda_{\omega i} f\right\|^{2} d \mu(\omega) \leqslant \\
\leqslant \sum_{i \in[m]}\left(\int_{\sigma_{i}}\left\|\Lambda_{\omega i} f\right\|^{2} d \mu(\omega)+\sum_{\substack{j \in[m] \\
j \neq i}} \int_{\sigma_{j}}\left\{\left\|\Lambda_{\omega i} f-\Lambda_{\omega j} f\right\|^{2} d \mu(\omega)+\left\|\Lambda_{\omega j} f\right\|^{2} d \mu(\omega)\right\}\right) \leqslant \\
\leqslant \sum_{i \in[m]}\left(\int_{\sigma_{i}}\left\|\Lambda_{\omega i} f\right\|^{2} d \mu(\omega)+\sum_{\substack{j \in[m] \\
j \neq i}} \int_{\sigma_{j}}(M+1)\left\|\Lambda_{\omega j} f\right\|^{2} d \mu(\omega)\right)= \\
=\{(m-1)(M+1)+1\} \sum_{i \in[m]} \int_{\sigma_{i}}\left\|\Lambda_{\omega i} f\right\|^{2} d \mu(\omega) .
\end{gathered}
$$

This completes the proof.

\section{Construction of c-K-g-woven with Bounded Operators.} For a given c-K-g-woven $\left\{\Lambda_{\omega i} \in \mathcal{B}\left(H, H_{\omega}\right)\right\}_{\omega \in \Omega, i \in[m]}$ of $H$, we will obtain another c-K-g-woven for space. One approach is to construct the family $\left\{\Lambda_{\omega i} \in \mathcal{B}\left(H, H_{\omega}\right)\right\}_{\omega \in \Omega, i \in[m]}$, where $U \in \mathcal{B}(H)$. The following theorem gives us necessary and sufficient conditions for $\left\{\Lambda_{\omega i} U^{*}\right\}_{\omega \in \Omega, i \in[m]}$ to be a c-K-g-woven of $H$.

Theorem 5. Let $K \in \mathcal{B}(H)$ be a closed range, $\left\{\Lambda_{\omega i}\right\}_{\omega \in \Omega, i \in[m]}$ be a $c$-K-g-woven for $H$ with universal bounds $A, B$ and $U \in \mathcal{B}(H)$ with $\mathcal{R}\left(U^{*}\right) \subseteq \mathcal{R}(K)$. Then $\left\{\Lambda_{\omega i} U^{*}\right\}_{\omega \in \Omega, i \in[m]}$ is a $c$-K-g-woven for $H$ if and only if there exists a constant $\delta>0$, such that for all $f \in H$

$$
\left\|U^{*} f\right\| \geqslant \delta\left\|K^{*} f\right\| \text {. }
$$

Proof. First, since $U^{*} f \in H$ and $\omega \mapsto\left\|\Lambda_{\omega i} f\right\|$ is a measurable function for each $f \in H$ and $i \in[m]$, then $\omega \mapsto\left\|\Lambda_{\omega i} U^{*} f\right\|$ is measurable for any $f \in H$ and $i \in[m]$. Suppose that $\left\{\Lambda_{\omega i} U^{*}\right\}_{\omega \in \Omega, i \in[m]}$ to be a c-K-g-woven for $H$ with the lower bound $C$. If $B$ is the upper bound of $\left\{\Lambda_{\omega i}\right\}_{\omega \in \Omega, i \in[m]}$, then for each $f \in H$ and any partition $\left\{\sigma_{i}\right\}_{i \in[m]}$ of $\Omega$,

$$
C\left\|K^{*} f\right\|^{2} \leqslant \sum_{i \in[m]} \int_{\sigma_{i}}\left\|\Lambda_{\omega i} U^{*} f\right\|^{2} d \mu \leqslant B\left\|U^{*} f\right\|^{2} .
$$


Hence, $\left\|U^{*} f\right\| \geqslant \sqrt{\frac{C}{B}}\left\|K^{*} f\right\|$. As for the opposite implication, for every $f \in H$ we have

$$
\left\|U^{*} f\right\|=\left\|\left(K^{\dagger}\right)^{*} K^{*} U^{*} f\right\| \leqslant\left\|K^{\dagger}\right\| \cdot\left\|K^{*} U^{*} f\right\| .
$$

Therefore, for each partition $\left\{\sigma_{i}\right\}_{i \in[m]}$ of $\Omega$

$$
\begin{aligned}
A \delta^{2}\left\|K^{\dagger}\right\|^{-2}\left\|K^{*} f\right\|^{2} \leqslant A\left\|K^{\dagger}\right\|^{-2}\left\|U^{*} f\right\|^{2} \leqslant A\left\|K^{*} U^{*} f\right\|^{2} & \leqslant \\
& \leqslant \sum_{i \in[m]} \int_{\sigma_{i}}\left\|\Lambda_{\omega i} U^{*} f\right\|^{2} d \mu .
\end{aligned}
$$

The upper bound condition is given similarly.

Corollary. Let $K$ be a closed range, $\left\{\Lambda_{\omega i}\right\}_{\omega \in \Omega, i \in[m]}$ be a c-K-g-woven for $H$ with universal bounds $A, B$. If $U \in \mathcal{B}(H)$ with $\mathcal{R}\left(U^{*}\right) \subseteq \mathcal{R}(K)$, then $\left\{\Lambda_{\omega i} U^{*}\right\}_{\omega \in \Omega, i \in[m]}$ is a $c-K$ - $g$-woven for $H$ if and only if $U$ is surjective.

Proof. By Theorem 5, we get, for any $f \in H$,

$$
\delta\left\|K^{\dagger}\right\|^{-1}\|f\| \leqslant \delta\left\|K^{*} f\right\| \leqslant\left\|U^{*} f\right\|
$$

and this completes the proof.

Theorem 6. Suppose that $\Lambda=\left\{\Lambda_{\omega i}\right\}_{\omega \in \Omega, i \in[m]}$ is a $c-K$-g-woven for $H$ with the lower bound $A$ and $U \in \mathcal{B}(H)$ with $\mathcal{R}(U) \subseteq \mathcal{R}(K)$. Then $\Lambda$ is a $c-U$-g-woven for $H$.

Proof. Due to Lemma 2, there exists $\alpha>0$, such that $U U^{*} \leqslant \alpha^{2} K K^{*}$. Let $\left\{\sigma_{i}\right\}_{i \in[m]}$ be a partition of $\Omega$. Then, for each $f \in H$, we have

$$
A \alpha^{-2}\left\|U^{*} f\right\|^{2} \leqslant A\left\|K^{*} f\right\|^{2} \leqslant \sum_{i \in[m]} \int_{\sigma_{i}}\left\|\Lambda_{\omega i} f\right\|^{2} d \mu(\omega) .
$$

Thus, $\Lambda$ is a c- $U$-g-woven.

Theorem 7. Suppose that $K \in \mathcal{B}(H)$ has dense range, $\Lambda=\left\{\Lambda_{\omega i}\right\}_{\omega \in \Omega, i \in[m]}$ is a $c-K$ - $g$-woven for $H$, and $U \in \mathcal{B}(H)$ has closed range. If $\left\{\Lambda_{\omega i} U^{*}\right\}_{\omega \in \Omega, i \in[m]}$ is a $c-K$ - $g$-woven for $H$, then $U$ is surjective.

Proof. Assume that $\left\{\sigma_{i}\right\}_{i \in[m]}$ is a partition of $\Omega$. Hence, for any $f \in H$, we have

$$
A\left\|K^{*} f\right\|^{2} \leqslant \sum_{i \in[m]} \int_{\sigma_{i}}\left\|\Lambda_{\omega i} U^{*} f\right\|^{2} d \mu(\omega) .
$$


Since $\mathcal{N}\left(K^{*}\right)=\mathcal{R}(K)^{\perp}$ and $K$ has dense range, $K^{*}$ is injective. By (3), $\mathcal{N}\left(U^{*}\right) \subseteq \mathcal{N}\left(K^{*}\right), U^{*}$ is injective. Moreover $\mathcal{R}(U)=\mathcal{N}\left(U^{*}\right)^{\perp}=H$. Thus, $U$ is surjective.

Corollary. Let $K \in \mathcal{B}(H)$ be a dense range, $\Lambda=\left\{\Lambda_{\omega i}\right\}_{\omega \in \Omega, i \in[m]}$ be a $c$-K-g-woven for $H$, and $U \in \mathcal{B}(H)$ be a closed range with $\mathcal{R}\left(U^{*}\right) \subseteq \mathcal{R}(K)$. Then $\left\{\Lambda_{\omega} U^{*}\right\}_{\omega \in \Omega}$ is a $c-K$-g-woven for $H$ if and only if $U$ is surjective.

Theorem 8. Suppose that $K \in \mathcal{B}(H)$ and $\Lambda=\left\{\Lambda_{\omega i}\right\}_{\omega \in \Omega, i \in[m]}$ is a $c$-K-g-woven for $H$ with the frame bounds $A, B$. If $U \in \mathcal{B}(H)$ has a closed range with $U K=K U$ and $\mathcal{R}\left(K^{*}\right) \subset \mathcal{R}(U)$, then $\left\{\Lambda_{\omega i} U^{*}\right\}_{\omega \in \Omega, i \in[m]}$ is a $c-K$-g-woven for $H$ with frame bounds $A\left\|U^{\dagger}\right\|^{-2}$ and $B\|U\|^{2}$.

Proof. As $U$ has closed range, it has the pseudo-inverse $U^{\dagger}$, such that $U U^{\dagger}=I$. Now, for each $f \in H$, we obtain

$$
\left\|K^{*} f\right\|^{2}=\left\|U U^{\dagger} K^{*} f\right\|^{2}=\left\|\left(U^{\dagger}\right)^{*} U^{*} K^{*} f\right\|^{2} \leqslant\left\|U^{\dagger}\right\|^{2}\left\|K^{*} U^{*} f\right\|^{2},
$$

from which we arrive at

$$
\sum_{i \in[m]} \int_{\sigma_{i}}\left\|\Lambda_{\omega i} U^{*} f\right\|^{2} d \mu(\omega) \geqslant A\left\|K^{*} U^{*} f\right\|^{2} \geqslant A\left\|U^{\dagger}\right\|^{-2}\left\|K^{*} f\right\|^{2} .
$$

The upper bound is obvious.

Theorem 9. Let $K \in \mathcal{B}(H)$ be a dense range, $\Lambda=\left\{\Lambda_{\omega i}\right\}_{\omega \in \Omega, i \in[m]}$ be a $c$-K-g-woven for $H$, and $U \in \mathcal{B}(H)$ be a closed range. If $\left\{\Lambda_{\omega i} U^{*}\right\}_{\omega \in \Omega, i \in[m]}$ and $\left\{\Lambda_{\omega i} U\right\}_{\omega \in \Omega, i \in[m]}$ are $c-K$-g-woven for $H$, then $U$ is invertible.

Proof. Suppose that $A_{1}, B_{1}$ are the universal bounds of $\left\{\Lambda_{\omega i} U^{*}\right\}_{\omega \in \Omega, i \in[m]}$ and $A_{2}, B_{2}$ are the universal bounds of $\left\{\Lambda_{\omega i} U\right\}_{\omega \in \Omega}$. For any $f \in H$ we have

$$
A_{1}\left\|K^{*} f\right\|^{2} \leqslant \sum_{i \in[m]} \int_{\sigma_{i}}\left\|\Lambda_{\omega i} U^{*} f\right\|^{2} d \mu(\omega)\left\|^{2} d \mu \leqslant B_{1}\right\| f \|^{2}
$$

and $\mathcal{N}\left(U^{*}\right) \subseteq \mathcal{N}\left(K^{*}\right)$. Since $K$ has dense range, $K^{*}$ is injective. Moreover, $\mathcal{R}(U)=\mathcal{N}\left(U^{*}\right)^{\perp}=H$, then $U$ is surjective. Also, by

$$
A_{2}\left\|K^{*} f\right\|^{2} \leqslant \sum_{i \in[m]} \int_{\sigma_{i}}\left\|\Lambda_{\omega i} U f\right\|^{2} d \mu \leqslant B_{2}\|f\|^{2},
$$

we get $\mathcal{N}(U) \subset \mathcal{N}\left(K^{*}\right)$, so $U$ is injective. Thus, $U$ is invertible. 
Corollary. Suppose that $K \in \mathcal{B}(H)$ has a dense range, that $U \in \mathcal{B}(H)$ has a closed range with $U K=K U$, and $\Lambda_{\omega i} \in \mathcal{B}\left(H, H_{\omega}\right)$ for any $\omega \in \Omega$, $i \in[m]$. If $\left\{\Lambda_{\omega i} U\right\}_{\omega \in \Omega, i \in[m]}$ and $\left\{\Lambda_{\omega i} U^{*}\right\}_{\omega \in \Omega, i \in[m]}$ are both $c$-K-g-woven for $H$, then $\left\{\Lambda_{\omega i}\right\}_{\omega \in \Omega, i \in[m]}$ is a $c-K$-g-woven for $H$.

Proof. By theorem 9, $U$ is invertible on $H$. Since $U K=K U, U^{-1} K=$ $=K U^{-1}, \mathcal{R}\left(K^{*}\right) \subset \mathcal{R}\left(U^{-1}\right)=H$, and

$$
\left\{\Lambda_{\omega i}\right\}_{\omega \in \Omega, i \in[m]}=\left\{\Lambda_{\omega i} U^{*}\left(U^{-1}\right)^{*}\right\}_{\omega \in \Omega, i \in[m]} .
$$

Hence, by Theorem 8, $\left\{\Lambda_{\omega i}\right\}_{\omega \in \Omega, i \in[m]}$ is a c-K-g-woven for $H$.

Theorem 10. Let $\Lambda=\left\{\Lambda_{\omega i}\right\}_{\omega \in \Omega, i \in[m]}$ be a $c$-K-g-woven for $H$ and $U \in \mathcal{B}(H)$ be a co-isometry (i. e., $U U^{*}=1$ ) with $U K=K U$. Then $\left\{\Lambda_{\omega i} U^{*}\right\}_{\omega \in \Omega, i \in[m]}$ is a $c-K$-g-woven for $H$ with universal bounds $A$ and $B$.

Proof. Let $A, B$ be the universal bounds of $\Lambda$. Since $U$ is co-isometry, we have, for each $f \in H$,

$$
\sum_{i \in[m]} \int_{\sigma_{i}}\left\|\Lambda_{\omega i} U^{*} f\right\|^{2} d \mu(\omega) \geqslant A\left\|K^{*} U^{*} f\right\|^{2}=A\left\|U^{*} K^{*} f\right\|^{2}=A\left\|K^{*} f\right\|^{2}
$$

For the upper bound, it is clear that

$$
\sum_{i \in[m]} \int_{\sigma_{i}}\left\|\Lambda_{\omega i} U^{*} f\right\|^{2} d \mu(\omega) \leqslant B\left\|U^{*} f\right\|^{2}=B
$$

and the proof is completed.

4. Perturbation for $\mathbf{c - K - g - w o v e n . ~ B e m r o s e ~ e t ~ a l . ~ i n ~ [ 6 ] ~ p r o v e d ~}$ sufficient conditions for weaving frames using perturbation and diagonal dominance. Perturbation of c-K-g-frames and weaving c-g-frames has been investigated in $[3,5]$. We study a Paley-Wiener-type perturbation for c-K-g-woven.

Theorem 11. Let $\left\{\Lambda_{\omega}\right\}_{\omega \in \Omega}$ and $\left\{\Gamma_{\omega}\right\}_{\omega \in \Omega}$ be two c-K-g-frames for $H$ with frame bounds $A_{1}, B_{1}$ and $A_{2}, B_{2}$, respectively. Suppose that there exist non-negative scalers $\eta$ and $0 \leqslant \lambda<\frac{1}{2}$, such that $\left(\frac{1}{2}-\lambda\right) A_{1}>\gamma$, and for each $f \in H$,

$$
\int_{\Omega}\left\|\left(\Lambda_{\omega}-\Gamma_{\omega}\right) f\right\|^{2} d \mu(\omega) \leqslant \lambda \int_{\Omega}\left\|\Lambda_{\omega} f\right\|^{2} d \mu(\omega)+\gamma\left\|K^{*} f\right\|^{2} .
$$


Then, $\left\{\Lambda_{\omega}\right\}_{\omega \in \Omega}$ and $\left\{\Gamma_{\omega}\right\}_{\omega \in \Omega}$ are $c$-K-g-woven for $H$ with universal frame bounds $\left(\frac{1}{2}-\lambda\right) A_{1}-\gamma$ and $B_{1}+B_{2}$.

Proof. The upper frame bound is clear. For the lower frame bound, assume that $\sigma \subset \Omega$ and we get, by the arithmetic-quadratic mean, for any $f \in H$ :

$$
\begin{gathered}
\int_{\sigma}\left\|\Lambda_{\omega} f\right\|^{2} d \mu(\omega)+\int_{\sigma^{c}}\left\|\Gamma_{\omega} f\right\|^{2} d \mu(\omega) \geqslant \\
\geqslant \int_{\sigma}\left\|\Lambda_{\omega} f\right\|^{2} d \mu(\omega)+\frac{1}{2} \int_{\sigma^{c}}\left\|\Lambda_{\omega} f\right\|^{2} d \mu(\omega)-\int_{\sigma^{c}}\left\|\left(\Lambda_{\omega}-\Gamma_{\omega}\right) f\right\|^{2} d \mu(\omega)= \\
=\frac{1}{2} \int_{\Omega}\left\|\Lambda_{\omega} f\right\|^{2} d \mu(\omega)+\frac{1}{2} \int_{\sigma}\left\|\Lambda_{\omega} f\right\|^{2} d \mu(\omega)-\int_{\sigma^{c}}\left\|\left(\Lambda_{\omega}-\Gamma_{\omega}\right) f\right\|^{2} d \mu(\omega) \geqslant \\
\geqslant \frac{1}{2} \int_{\Omega}\left\|\Lambda_{\omega} f\right\|^{2} d \mu(\omega)-\int_{\sigma^{c}}\left\|\left(\Lambda_{\omega}-\Gamma_{\omega}\right) f\right\|^{2} d \mu(\omega) \geqslant \\
\geqslant \frac{1}{2} \int_{\Omega}\left\|\Lambda_{\omega} f\right\|^{2} d \mu(\omega)-\lambda \int_{\Omega}\left\|\Lambda_{\omega} f\right\|^{2} d \mu(\omega)-\gamma\left\|K^{*} f\right\|^{2} \geqslant \\
\geqslant\left(\left(\frac{1}{2}-\lambda\right) A_{1}-\gamma\right)\left\|K^{*} f\right\|^{2} .
\end{gathered}
$$

This completes the proof.

Acknowledgements. We gratefully thank the referee for carefully reading the paper and for the suggestions that have greatly improved the presentation of the paper.

\section{References}

[1] Abdollahpour M. R., Faroughi M. H. Continuous G-frames in Hilbert spaces, Southeast Asian Bull. Math., 2008, vol. 32, no. 1, pp. 1-19.

[2] Ali S. T., Antoine J. P., Gazeau J. P. Continuous frames in Hilbert spaces, Ann. Phys., 1993, vol. 222, no. 1, pp. 1-37.

[3] Alizadeh E., Rahimi A., Osgooei E., Rahmani M. Continuous K-g-frames in Hilbert spaces, Bull. Iranian Math. Soc., 2019, vol. 45, no. 4, pp. 10911104.

[4] Alizadeh E., Rahimi A., Osgooei E., Rahmani M. Some Properties of Continuous K-g-frames in Hilbert spaces, U.P.B. Sci. Bull., Series A, 2019, vol. 81 , no. 3 , pp. $43-52$. 
[5] Alizadeh E., Sadri V. On Continuous weaving G-frames in Hilbert spaces, Wavelets and Linear Algebra. Note, 2020, vol. 7, no. 1, pp. 23-36.

DOI: https://doi.org/10.22072/WALA.2020.114423.1248

[6] Bemrose T., Casazza P. G., Gröchenic K., Lammers M. C., Lynch R. G. Weaving frames, Operators and Matrices, 2016, vol. 10, no. 4, pp. 10931116.

[7] Casazza P. G. Kutyniok G. Frames of Subspaces. Contemp. Math.Anal., 2004, vol. 345, pp. 87-114.

[8] Casazza P. G., Lynch R. G. Weaving properties of Hilbert space frames, International Conference on Sampling Theory and Applications (SampTA)., 2015, pp. 110-114.

[9] Christensen O. An Introduction to Frames and Riesz Bases, Birkhäuser, Boston, 2016.

[10] Deepshikha and Vashisht L. K., On perturbation of local atoms for subspaces, Poincare J. Anal. Appl., 2015, 2, pp. 129-137.

[11] Dehghan M. A., Hasankhani Fard M. A. G-continuous frames and coorbit spaces, Acta Math. Acad. Paedagogicae Nyíregyháziensis. (N.S.), 2008, vol. 24, no. 3, pp. 373-383.

[12] Douglas R. G. No majorization, factorization and range inclusion of operators on Hilbert space, Pro. Amer. Math. Sco., 1966, vol. 17, no. 2, pp. 413415.

[13] Duffin R. J., Schaeffer A. C. A class of nonharmonic Fourier series. Trans. Amer. Math. Soc. Anal., 1952, vol. 72, no. 1, pp. 341-366.

[14] Faroughi M. H., Osgooei E. C-frames and c-Bessel mappings, Bull. Iranian Math. Soc., 2012, vol. 38, no. 1, pp. 203-222.

[15] Feichtinger H. G., Werther T. Atomic Systems for Subspaces. Proceedings SampTA. Orlando, FL Anal., 2001, pp. 163-165.

[16] Găvruţa L. Frames for Operators. Appl. Comp. Harm. Anal., 2012, vol. 32, pp. 139-144.

[17] Kaiser G. A Friendly Guide to Wavelets, Birkhäuser, Boston, 1994.

[18] Rahimlou Gh., Ahmadi R., Jafarizadeh M. A., Nami S. Continuous $K$-frames and their dual in Hilbert spaces, Sahand Comm. Math. Anal., 2020, vol. 17, no. 3, pp. 145-160.

[19] Sadri V., Rahimlou Gh., Ahmadi R., Zarghami Farfar R. Construction of K-g-fusion frames and their dual in Hilbert spaces, Bull. Transilvania Un. Braşov, Series III, 2020, vol. 13(62), no. 1, pp. 17-32. 
[20] Sadri V., Ahmadi R., Jafarizadeh M. A., Nami S. Continuous K-fusion frames in Hilbert spaces, Sahand Comm. Math. Anal., 2020, vol. 17, no. 1, pp. 39-55.

[21] Sun W. G-Frames and G-Riesz bases. J. Math. Anal. Appl., 2006, 326, pp. $437-452$.

[22] Vashisht L. K., Deepshikha On continuous weaving frames, Adv. Pure Appl. Math., 2017, vol. 8, no. 1, pp. 15-31.

Received November 5, 2020.

In revised form, February 18, 2021.

Accepted February 20, 2021.

Published online April 8, 2021.

Esmaeil Alizadeh

Department of Mathematics, Marand Branch, Islamic Azad University,

Marand, Iran

E-mail: e_alizadeh@marandiau.ac.ir

Vahid Sadri

Department of Mathematics, Faculty of Tabriz Branch, Technical and Vocational University (TVU), East Azarbaijan, Iran.

E-mail: vahidsadri57@gmail.com 